\title{
Trace Level Determination of Cadmium in Different Water Matrices and Rose Hip Tea Sample by Dispersive Liquid-liquid Microextraction Slotted Quartz Tube Flame Atomic Absorption Spectrometry After Complexation With a New Imidazole-based Ligand
}

\author{
Elif Yazicia ${ }^{a}$, Merve Firat ${ }^{a}$, Dotse Selali Chormey ${ }^{a}$, Türkan Börklü Budak ${ }^{a}$, Çigdem Sahin ${ }^{b}$, \\ Fatma Turak ${ }^{\mathrm{a}}$ and Sezgin Bakirdere ${ }^{\mathrm{a}, *}$
}

a Yildiz Technical University, Department of Chemistry, 34349 Istanbul, Turkey

b Pamukkale University, Faculty of Art and Science, Chemistry Department, 20070, Denizli, Turkey

\section{INTRODUCTION}

Rapid urbanization and an increase in the number of industries to meet the demands of an increasing population has lead to environmental contamination (1).

Cadmium (Cd) is a typical heavy metal known as a non-essential element for humans and an environmental contaminant resulting from anthropogenic activities such as mineral mining, ore smelting, sewage sludge, and industrial wastes (2). Cadmium enters the human body by inhalation of contaminated air, drinking contaminated water and liquids, ingesting foods, and skin contact with contaminated particles (3). Smoking is another source of cadmium entry into humans, and cigarettes are reported to contain about $1-2 \mu \mathrm{g}$ of cadmium (4). A major concern of cadmium contamination is its ability to cause severe health disorders of the kidneys, lungs, bones and liver, and some carcinogenic effects (5). According to the World Health Organization (WHO) and the U.S. Environmental Protection Agency (EPA), cadmium in drinking water should not exceed $3.0 \mu \mathrm{g} / \mathrm{L}$ and 5.0 $\mu \mathrm{g} / \mathrm{L}$, respectively (6). These allowable limits require sensitive analyti-

*Corresponding author.

E-mail: bsezgin23@yahoo.com

Tel: +90 2123834245

\section{ABSTRACT}

This study presents a sensitive, accurate, and precise analytical method for the determination of cadmium in water samples. A laboratory synthesized imidazolebased ligand (2-(4-methylphenyl)1H-imidazo-[4,5-f]-[1,10]-phenanthroline) was used to form a cadmium complex for extraction by dispersive liquid-liquid microextraction (DLLM). Optimization of complexation, extraction, and instrumental parameters led to a 74-fold enhancement in the detection power of FAAS. The limits of detection and quantification were found at 0.57 and 1.9 $\mu \mathrm{g} / \mathrm{L}$, respectively. The relative standard deviation of the method was less than $5.0 \%$, indicating high precision for the experimental process. Accuracy and applicability of the method were tested on environmental (tap, well, and wastewater) and drink (rose hip tea) samples at different spiking concentrations, and satisfactory recovery results were obtained between 88 and $112 \%$.

cal techniques for the accurate and precise determination of cadmium at trace levels.

Spectroscopic techniques have been widely used in the literature for the determination of cadmium and other elements in a variety of samples. Most of the analytical techniques are not suitable for direct analysis of the samples and, therefore, require sample treatment or processing prior to analyte determination (7). The atomic absorption spectrometry (AAS) techniques differ primarily based on their sample introduction and atomization units. In addition, the most common AAS systems such as flame (FAAS), hydride generation (HGAAS), photochemical vapor generation (PVGAAS), electrothermal (ETAAS) and cold-vapor (CVAAS) (8-12). HGAAS and PVG-AAS are selective for certain elements, offering high sensitivities that equals ETAAS and monitor only one metal at a time. However, inductively coupled plasma mass spectrometry and optical emission spectrometry (ICP-MS and ICP-OES) instruments perform simultaneous multi-elemental determinations but are much more costly (13). Solid samples usually require dissolution by acid digestion before instrumental readout, but techniques such as instrumental neutron activation analysis (INAA) and laser ablation inductively coupled plasma mass spectrometry (LA-ICP-MS) can be used for direct analysis of solid samples (14). FAAS is a widely used analytical technique for metal determination, but it has relatively low sensitivity due to poor sample nebulization efficiency. In the absence of the more sensitive instruments in a laboratory, preconcentration methods can be used to improve the detection power of the FAAS. 
Extraction is one the most used sample treatment methods for the separation of analytes from the sample matrix and preconcentration into measurable amounts. The classical liquid-liquid extraction and solid phase extraction methods have been replaced by microextraction methods which use less toxic solvents, produce high output, analyte recovery, and are rapid, economical, and easily automated (15). Dispersive liquid-liquid microextraction (DLLME) is one of the most common liquid phase microextraction strategies introduced for the determination of polycyclic aromatic hydrocarbons in aqueous samples. It has since been extensively used to extract both organic and inorganic analytes from a variety of matrices (16-18). DLLME is a fairly simple method which involves injecting a mixture of extraction and dispersive solvents into aqueous solutions. Due to the miscibility of the dispersive solvents with both aqueous solution and extraction solvent, the surface area for extraction is greatly increased which leads to high extraction efficiency and analyte recovery (18). In addition to the extraction methods, slotted quartz tubes (SQT) based on two working modes have also been used to improve the detection power of the FAAS system. In the first working mode, a slight increase of about 2-5 fold (depending on metal type) is obtained by simply placing the SQT on the flame unit to prevent a rapid exit of the atomized analyte, thereby increasing interaction with lamp radiation for high absorbance readings (19). The second working principle of SQT is based on the preconcentration by trapping the analyte atoms within the inner surface of the quartz tube for an optimum period of time. A sharp increase in flame temperature leads to a sudden release of the trapped atoms to obtain transient analytical signals (19).
The aim of this study was to develop an analytical strategy for cadmium based on DLLME-SQTFAAS after complexing with a synthesized imidazole ligand. The developed method was applied to different water matrices and rose hip tea sample to check its accuracy and applicability.

\section{EXPERIMENTAL}

\section{Solution Standards and Reagents}

Analytical grade reagents were used throughout the study. The working solutions of cadmium were prepared by volumetric dilution of the aliquots from a 1000$\mathrm{mg} / \mathrm{L}$ stock standard solution of cadmium purchased from High Purity Standards (Charleston, SC, USA). Deionized water with $18.2 \Omega$.cm resistivity was obtained from an ultrapure water purification system (Milli-Q ${ }^{\circledR}$, Millipore Corporation, USA). The ligand used for cadmium complexation, 2-(4-methylphenyl)-1H-imidazo[4,5-f]-[1,10]-phenanthroline (phenme), was synthesized in the laboratory and dissolved in methanol to prepare a $0.2 \%(\mathrm{w} / \mathrm{v})$ stock solution. Dilute acid/base adjusters were used to lower or increase the $\mathrm{pH}$ (between 5.0 to 11.0) of the primary salt solutions (potassium dihydrogen phosphate, di-sodium tetraborate decahydrate, and sodium bicarbonate). Other chemicals and reagents used in the method development process were

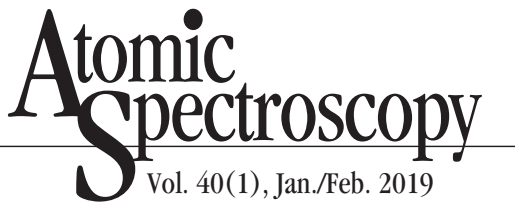

potassium chloride, potassium nitrate, sodium chloride, methanol, 2-propanol, ethanol, chloroform, 1,2-dichloroethane and carbon tetrachloride, all obtained from Merck (Germany).

\section{Instrumentation}

An Analytik Jena model NovAA 300 atomic absorption spectrometer with a flame unit was used for cadmium determination at the $228.8 \mathrm{~nm}$ analytical line. The cadmium hollow cathode lamp was operated at a current of $2.0 \mathrm{~mA}$ and a spectral bandpass of $0.50 \mathrm{~nm}$, a deuterium lamp $\left(\mathrm{D}_{2}\right)$ was used for background corrections. An acetylene/air mixture was used to form the flame, and sample introduction to the flame was achieved with the conventional nebulizer unit with optimized flow rates. A $5.5-\mathrm{cm}$ entry slot was made on a $13-\mathrm{cm}$ long quartz tube in order for the burner flame $(5.0 \mathrm{~cm})$ to enter the tube conveniently. An exit slot (3.0 $\mathrm{cm})$ was cut on the upper overlaying side of the entry slot. The internal and diameter lengths of the quartz tube were 16 and $18 \mathrm{~mm}$, respectively. A schematic diagram of the FAAS-SQT system is presented in Figure 1. A Spectrum Two FT-IR spectrometer (PerkinElmer, Inc., Shelton, CT, USA) with a diamond ATR and a VNMRJ 400 NMR instrument (Varian, USA) were used to record the infrared (IR) and nuclear magnetic resonance (NMR) spectra of the ligand, respectively.

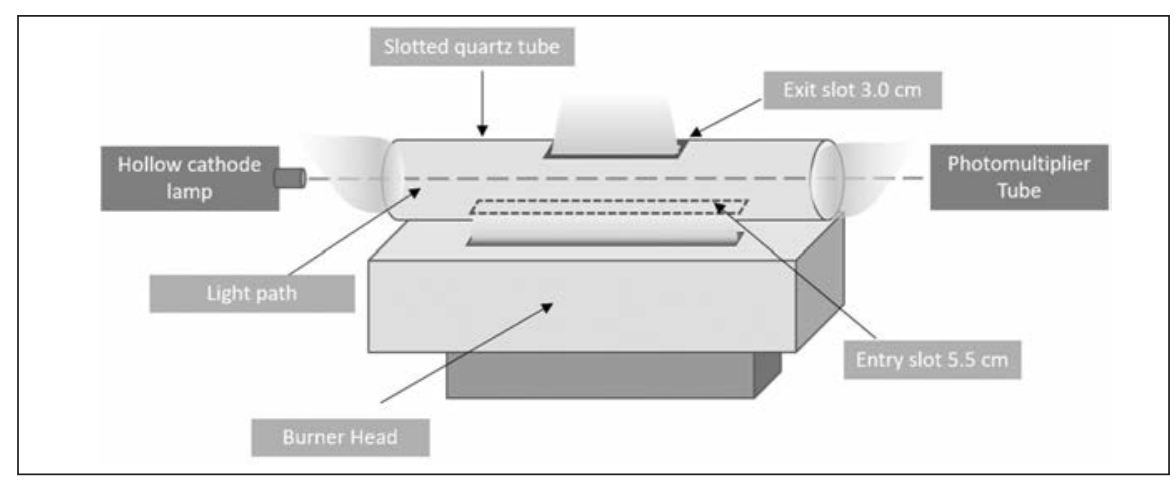

Fig. 1. Schematic diagram of SQT-FAAS system (20). 


\section{Samples}

Well water was sampled from the Anatolian region in Turkey, placed into clean polypropylene plastic containers, and stored at room temperature. Municipal wastewater from a biological treatment plant was sampled and stored at about $4.0^{\circ} \mathrm{C}$. Both samples were filtered through $125-\mathrm{mm}$ Whatman filter papers and then through 0.45 $\mu \mathrm{m}$ Teflon ${ }^{\circledR}$ syringe filters. Tap water (obtained from the analytical laboratory) was directly sampled into the clean tubes and used for the recovery studies. A rose hip sample was purchased at a local market in Istanbul. In the preparation of the tea sample, about $20 \mathrm{~g}$ of rose hip was weighed into a beaker and $150 \mathrm{~mL}$ of deionized water was added. The sample was heated to boiling point and allowed to boil for 15 minutes, then the sample was cooled and filtered through a $125-\mathrm{mm}$ filter paper.

Synthesis of 2-(4-methylphenyl) -1H-imidazo-[4,5-f]-[1,10]phenanthroline

A procedure from literature was slightly modified and used to prepare and purify 2-(4-methylphenyl)1H-imidazo-[4,5-f]-[1,10]-phenanthroline (21). 1,10-phenanthroline5,6-dione was prepared according to the procedure by Zhong et al. (22). The following mixture was refluxed under an argon atmosphere for 8 hours: p-tolualdehyde (5.14 mmol), 1,10-phenanthroline5,6-dione (4.28 mmol), ammonium acetate $(85.6 \mathrm{mmol})$, and glacial acetic acid $(50 \mathrm{~mL})$. The solution was cooled to ambient temperature, then diluted with distilled water, and neutralized with a solution of ammonia. Yellow precipitates were gathered and washed with distilled water. An $87 \%$ yield of dried product was obtained after dissolution of the precipitate in dichloromethane, and re-precipitation with diethyl ether.

\section{Dispersive Liquid-liquid Microextraction}

The cadmium-phen-me complex was formed by adding $0.50 \mathrm{~mL}$ of $\mathrm{pH}$ buffer solution and $1.0 \mathrm{~mL}$ of ligand to $8.0 \mathrm{~mL}$ aqueous standard/ sample solution to a 15 -mL centrifuge tube. The tube was sealed and shaken slightly to uniformly spread the ligand throughout the aqueous solution. $3.0 \mathrm{~mL}$ of methanol and $0.40 \mathrm{~mL}$ of chloroform were mixed in a separate tube, withdrawn with a 5.0-mL syringe, then injected into the complex solution. The solution was vortexed for 15 seconds and placed into a centrifuge for $2.0 \mathrm{~min}$ utes at $3461 \mathrm{~g}$. The aqueous phase was decanted and the bottom complex organic phase extracted was transferred into a clean tube and completely evaporated at about $100^{\circ} \mathrm{C}$. Concentrated nitric acid was used to dissolve the residue and the final solution was analyzed in the SQT-FAAS system.

\section{RESULTS AND DISCUSSION}

A systematic optimization of parameters was carried out with 50 $\mu \mathrm{g} / \mathrm{L}$ aqueous standard solution of cadmium, where one parameter was optimized at a time while keeping others fixed. The highest mean absorbance from three replicate measurements was used to select optimum parameters. The synthesized ligand was characterized by H-NMR and FTIR as presented in the results below:

${ }^{1} \mathrm{H}$ NMR $\left(\mathrm{CDCl}_{3}\right) \partial \mathrm{ppm}: 8.83$ (s, $4 \mathrm{H}), 8.13(\mathrm{~d}, 2 \mathrm{H}), 7.41(\mathrm{~s}, 2 \mathrm{H})$, $7.1(\mathrm{~d}, 2 \mathrm{H}), 2.29(\mathrm{~s}, 3 \mathrm{H})$

FTIR (ATR, $\mathrm{cm}^{-1}$ ): 3345, 30203040, 2880-2920, 1605, 1550, 1482, $1448,1348,825,736,620$

\section{Complex Formation Optimization}

There are few studies in literature that use ligandless extraction methods for metals $(23,24)$. Complexation with an appropriate lig- and is a common practice that allows the extraction of metals from aqueous solution with an organic solvent. The efficiency of complexation is very important as it relates to the extraction yield. Thus, complexation conditions such as the $\mathrm{pH}$ of the buffer and the ligand concentration were optimized to obtain high output. The buffer solutions at $\mathrm{pH}$ of 5.0, 6.0, $7.0,8.0,9.0,10$, and 11 were added to the aqueous standards before ligand addition to determine the correct $\mathrm{pH}$ balance for the formation of the cadmium-phen-me complex. All buffer solutions were extracted and measured under the same conditions, and the absorbance values increased from $\mathrm{pH} 5.0$ to 9.0, which then became constant. The buffer at $\mathrm{pH} 9$ was selected as optimum due to its relatively high precision (exact \%RSD value) for replicate measurements. The $\mathrm{pH} 9$ was tested for buffer solutions of $0.50,1.0,1.5$, and $2.0 \mathrm{~mL}$ volumes. It was found that the absorbance values obtained were not significantly different from each other. Therefore, $0.50 \mathrm{~mL}$ was selected because it presented the least dilution to the analyte solution.

The integrity of the prepared ligand solution was ensured by keeping it in a cool dry place and wrapped in aluminum foil against sunlight and light degradation. From the $0.20 \%$ stock solution, $0.10,0.05$, and $0.02 \%$ concentrations were prepared by dilution and all four concentrations $(0.02$, $0.05,0.10$, and $0.20 \%$ ) were tested for their complexation output. $0.05 \%$ was selected for further studies because the absorbance signals only increased marginally with an increasing ligand concentration. In order to ensure an even distribution and to increase the rate of complexation, the standard solutions were subjected to different time periods ( 0.25 - 90 minutes) of mechanical shaking. The mechanical shaking ensured homogenous 
mixing, but the absorbance output recorded decreased with an increase in mixing periods. It was found that 15 seconds of mechanical mixing after ligand addition offered the best results .

\section{Extraction Solvent Type and Amount}

Four organic solvents with densities greater than 1.0 (chloroform, carbon tetrachloride, dichloromethane, and 1,2-dichloroethane) were selected and tested to check their extraction power for the cadmium complex. These solvents were selected based on their high densities in order to obtain clearly separated organic phases from the aqueous solution at the bottom of the conical tubes used. An amount of $350 \mu \mathrm{L}$ of each organic solvent was mixed with $3.0 \mathrm{~mL}$ ethanol and used to extract the cadmium standard solutions. No phase separation was observed for dichloromethane which can be attributed to the dispersive solvent further enhancing its solubility in water. The other solvents had distinct phases, but the volume of the settled phase decreased according to the percent solubilities. Carbon tetrachloride recorded the least absorbance value and chloroform was slightly higher than 1,2-dichloroethane. Hence, chloroform was selected, and its optimum amount was tested between 200 and $450 \mu \mathrm{L}$ at $50 \mu \mathrm{L}$ intervals. The absorbance values were observed to increase from $200 \mu \mathrm{L}$ up to $400 \mu \mathrm{L}$, after which it decreased slightly as shown in Figure 2. This suggested that a minimum of $400 \mu \mathrm{L}$ chloroform is required to collect a substantive amount of cadmium-phen-me complex from the aqueous solution.

\section{Dispersive Solvent Type and Amount}

The basic role of a dispersive solvent is to finely distribute extraction solvents throughout the aqueous solution, thereby increasing the surface area for efficient extraction. This can only be achieved by using solvents that are miscible with both the extraction solvent and the aqueous solution. Methanol, ethanol, and 2-propanol, which were identified to meet these criteria, were tested for their dispersion efficiencies. $3.0 \mathrm{~mL}$ of each solvent was mixed with $400 \mu \mathrm{L}$ chloroform and injected into similar aqueous standards. The absorbance values recorded for methanol were higher and more precise than the other dispersive solvents. The amount of dispersive solvent also affects the degree of extractant dispersion through the aqueous solution. A high amount could lead to over-

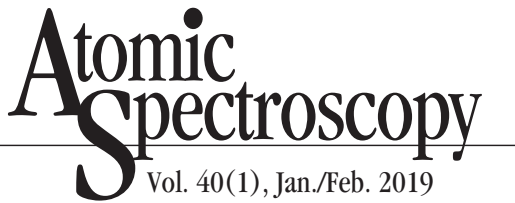

dispersion of the extractant and a low amount might not disperse the extractant enough to obtain the required surface area for efficient extraction. For this reason, the volumes of 4.0, 3.0, 2.0, and $1.0 \mathrm{~mL}$ of methanol were tested to obtain the optimum chloroform amount. Low absorbance values were recorded for 1.0 and $2.0 \mathrm{~mL}$ amounts, suggesting insufficient dispersion. The $4.0 \mathrm{~mL}$ methanol also recorded low absorbance values with respect to $3.0 \mathrm{~mL}$ and could have resulted from over-dispersion of chloroform, leading to a low extracted phase volume. The highest absorbance signal was recorded for $3.0 \mathrm{~mL}$ of methanol which was selected for further optimization studies.

\section{Salting-out and Mixing Effect}

Salts can be added to aqueous solutions during extraction procedures to increase the extraction output of the analytes. The added salt causes a phasing out effect of the analyte due to its high solubility, and separation of the phases after extraction can also be enhanced (25). Excess salt amounts could, however, make the solution very dense and limit the mass transfer of the analyte into the extraction solvent. A $0.10 \mathrm{~g}$ amount of potassium chloride, potassium nitrate, and sodium chloride was

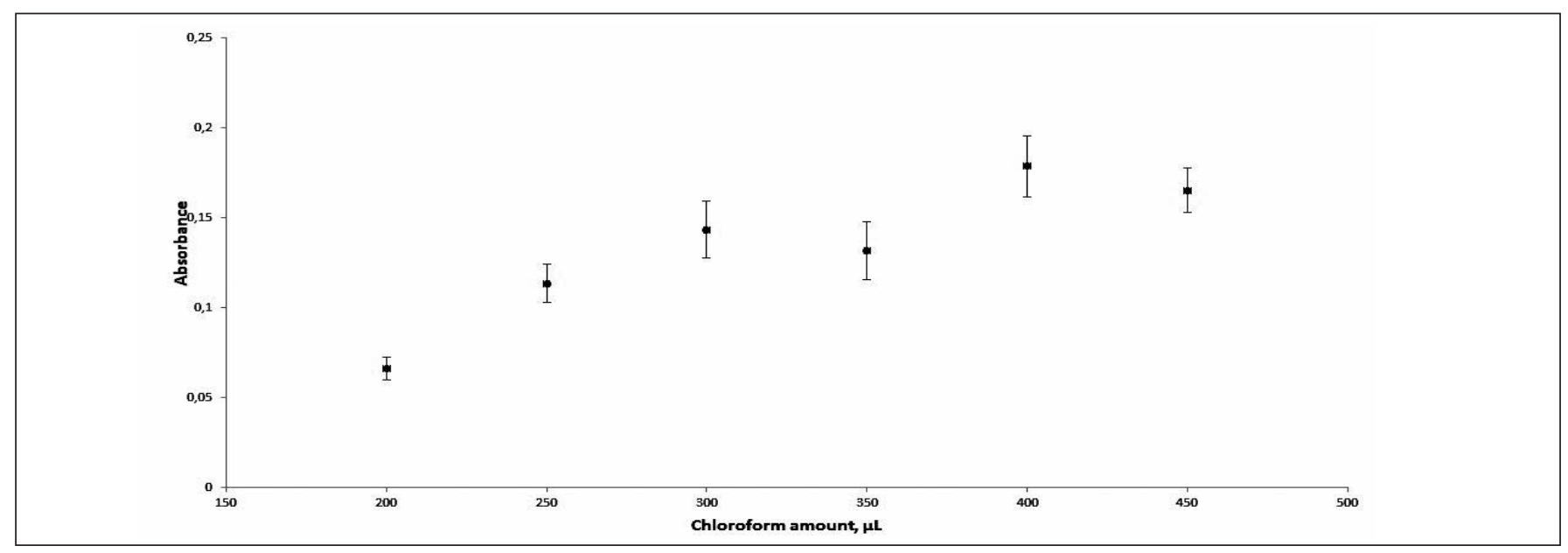

Fig. 2. A plot of chloroform amounts against absorbance $(n=3)$. 
tested and compared to a saltless extraction under the same conditions. All salt-added extractions resulted in lower absorbance signals relative to the saltless extraction. All other extractions were then performed without adding salt to the solution.

After injection of the extractant and disperser mixture into the aqueous solution, mixing by mechanical shaking, vortexing, manual shaking, and ultrasonication were tested to determine whether extraction output would be enhanced or not. Each mixing type was performed for 1.0 minute and from their absorbance values, manual shaking (up-down motion) had a significant impact on the extraction output. The optimum manual shaking period was determined by testing periods between 15 seconds and 5.0 minutes. The manual shaking time of 5.0 minutes was used to determine whether a very significant increase in comparison to prolonged periods. The absorbance results obtained showed that mixing beyond 30 seconds did not have any extra effect on the extraction output. Thus, 30 seconds of manual shaking was selected as the optimum mixing period.

\section{Analytical Figures of Merit \\ Calibration standards in wide} range were analyzed under the optimum experimental conditions listed in Table I. The analytical figures of merit were determined from the linear calibration plots developed. The limit of detection (LOD), limit of quantification (LOQ), precision (\%RSD), dynamic range, and coefficient of the determination (R2) were used to validate the method (see Table II). The optimum method for DLLME-SQT-FAAS was found applicable over a broad concentration range with good linearity $\left(R^{2}=0.9993\right)$ and high precision for the lowest concentration.
Using the limits of detection of each system under study, enhancement in instrumental detection power was calculated with respect to the conventional FAAS. The SQTFAAS, DLLME-FAAS, and DLLMESQT-FAAS systems were about 4.0, 21 , and 74 times more sensitive than the FAAS system. The LOD and LOQ values obtained in this study fall below the allowable limit of cadmium in drinking water set by WHO and the U.S. EPA. The LOD of this method $(0.57 \mu \mathrm{g} / \mathrm{L})$ is comparable to other methods in the literature that were used to preconcentrate cadmium for the determination by FAAS. The detection limits reported for cadmium using vortex-assisted ionic liquid-based liquid-liquid microextraction technique (VALLME), silica gel adsorbent and flow injection (FI), and fabric fiber sorbent extraction (FFSE) were $2.9,4.3$, and $0.30 \mu \mathrm{g} \mathrm{L}^{-1}$, respectively (26-28).

\section{Recovery Tests for the Samples}

The mineral content of drinking water could cause a hindrance to complexation and extraction of cadmium. Wastewater also has a very complicated matrix which could have varying effects on the extraction and determination of an analyte. In order to determine the applicability of this method to tap, well, and wastewater, recovery studies were performed by spiking the samples at different concentrations. Prior to spiking, blank determinations of the samples under optimum conditions were performed. No absorbance signal was recorded for all three blank determinations, and this implied that cadmium was not present or below the detection limit. Table III presents the recovery results obtained for the water samples spiked at $\mathbf{1 0}$, 20 , and $40 \mu \mathrm{g} / \mathrm{L}$ final concentration. Appreciable recovery results were obtained for all water samples, but

TABLE I

Optimized Parameters for the DLLME-SQT-FAAS Method

\begin{tabular}{ll}
\hline Parameter & Value \\
\hline pH of buffer solution & pH $9(0.50 \mathrm{~mL})$ \\
Ligand (concentration/amount) & Phen-me $(0.05 \%, \mathrm{w} / \mathrm{v} / 1.0 \mathrm{~mL})$ \\
Mixing Period (complex formation) & $15 \mathrm{~s}$ \\
Extraction solvent (amount) & Chloroform $(400 \mu \mathrm{L})$ \\
Dispersive solvent (amount) & Methanol $(3.0 \mathrm{~mL})$ \\
Mixing type (period) & Manual shaking $(30 \mathrm{~s})$ \\
Sample flow rate* & $7.2 \mathrm{~mL} / \mathrm{min}$ \\
Acetylene flow rate* & $40 \mathrm{~L} / \mathrm{h}$ \\
SQT height* & $2.0 \mathrm{~mm}$ \\
\hline
\end{tabular}

*Parameters obtained from Firat et al. (19) .

TABLE II

Analytical Performance of the FAAS and Upgrade of Systems Under Study

\begin{tabular}{lcccc}
\hline System & LOD $(\mu \mathrm{g} / \mathrm{L})$ & LOQ $(\mu \mathrm{g} / \mathrm{L})$ & RSD $(\%)$ & Range $(\mu \mathrm{g} / \mathrm{L})$ \\
\hline FAAS $(19)$ & 42 & 140 & 9.6 & $100-2000$ \\
SQT-FAAS & 12 & 40 & 6.3 & $50-1000$ \\
DLLME-FAAS & 2.1 & 6.7 & 8.2 & $7.5-125$ \\
DLLME-SQT-FAAS & 0.57 & 1.9 & 4.2 & $2.0-50$ \\
\hline
\end{tabular}


wastewater recorded relatively lower recovery results, presumably due to the complex nature of the waste matrices. These recovery results establish that cadmium when present can be complexed, extracted, and determined by the present method with a good level of accuracy and precision.

In order to check the applicability of the developed method in rose hip extract (tea), recovery studies based on spiking experiments were performed. Two brewed rose hip tea samples were cooled and filtered into clean polypropylene tubes. Triplicate blank determinations were performed for each sample under the optimum extraction conditions. A distinct bottom phase was not obtained after centrifugation, but the solid sediments were collected at the bottom of the tube even though precipitation was not observed upon addition of the ligand. To ensure that the sediments were not a result of precipitation, the sample solution was directly extracted with chloroform without adding the ligand and buffer solutions. The solid sediments occurred again and this suggested that minute rose hip particles were gathered at the bottom of the tube. The tea samples were, therefore, diluted 10 times and the solid sediments were eliminated. No analytical signals were recorded for the blank measurements, signifying cadmium was not present or below the detection limit. One of the tea samples was also spiked at 10, 40, and $50 \mu \mathrm{g} / \mathrm{L}$, and their percent recoveries determined using aqueous calibration standards. The absorbance values obtained for the spiked tea samples were about half the values obtained for the aqueous standards, leading to percent recoveries below $50 \%$. This is due to the complex matrix of the tea samples, but in order to overcome the matrix effect, matrix matching strategy was applied. Matrix matching was tested to make sure whether or not the recovery results could be improved upon. For this purpose, calibration standards used in the quantifications were similarly prepared in another tea sample. Prior to preparation of standards, blank determination was performed under the optimum conditions to determine the presence or absence of cadmium in the tea extract. There was no signal observed for tea sample extract which would be used in the preparation of the standards. A matrix-matched calibration plot was used to determine the percent recovery. The percent recoveries calculated for 10, 40, and 50 $\mu \mathrm{g} / \mathrm{L}$ spiked samples were found to be $103.3 \pm 1.3 \%, 107.7 \pm 3.8 \%$, and $95.6 \pm 2.8 \%$, respectively.

\section{CONCLUSION}

In this study, a sensitive, accurate, and precise analytical method was developed for the determination of cadmium at trace levels in water samples and in the extract of rose hip tea. A laboratory synthesized ligand was used to form a coordinate covalent complex of cadmium for the extraction by dispersive liquid-liquid microextraction (DLLME). The entire method was optimized to enhance the FAAS measurement sensitivity by 74 -fold.

TABLE III

Percent Recoveries of Well, Tap, and Wastewater Samples

\begin{tabular}{lccc}
\hline Sample & $10 \mu \mathrm{g} / \mathrm{L}(\%)$ & $20 \mu \mathrm{g} / \mathrm{L}(\%)$ & $40 \mu \mathrm{g} / \mathrm{L}(\%)$ \\
\hline Tap water & $112.1 \pm 3.3$ & - & $103.0 \pm 3.1$ \\
Well Water & $101.3 \pm 12.0$ & $105.0 \pm 1.3$ & $98.6 \pm 1.6$ \\
Wastewater & $100.6 \pm 3.0$ & $88.2 \pm 0.7$ & $88.6 \pm 2.6$ \\
\hline
\end{tabular}

Uncertainties $( \pm)$ are shown by standard deviation $(n=3)$.

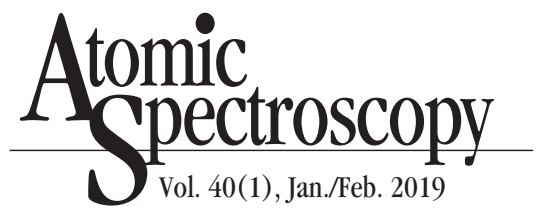

The detection limit obtained with this enhancement was $0.57 \mu \mathrm{g} / \mathrm{L}$ and the repeatability of the method was established by low percent relative standard deviation $(<5.0 \%)$. The figures of merit used to validate the method were satisfactory. The method's applicability was tested on well, tap, and wastewater as well as to a rose hip extract at different concentrations, and appreciable recovery results were obtained. The matrix matching strategy was applied to improve the accuracy of the method for the rose rip tea extract matrix. The method is simple, accurate, and low cost. The method is also rapid and in agreement with green chemistry since it uses low volumes of solvents.

Received July 23, 2018.

\section{REFERENCES}

1. Y.K. Li, T. Yang, M.L. Chen and J.H. Wang, Talanta 180, 18 (2018).

2. P. Rojjanateeranaj, C. Sangthong and B. Prapagdee, Chemosphere 185, 764 (2017)

3. M. Nordberg, In: Encyclopedia of Food Sciences and Nutrition (B. Caballero, ed.) (Second Edition), Academic Press, Oxford, UK, 739 (2003).

4. L. Järup and A. Åkesson, Toxicol. Appl. Pharmacol. 283, 201 (2009).

5. A.A. Tinkov, T. Filippini, O.P. Ajsuvakova, J. Aaseth, Y.G. Gluhcheva and J.M. Ivanova, Sci. Total Environ. 601-162, 741 (2017).

6. G.F. Nordberg, K. Nogawa, M. Nordberg and L.T. Friberg, In; Handbook on the Toxicology of Metals (Third Edition) Burlington: Academic Press, 445 (2007).

7. S.C. Moidoveanu and V. David, In: Journal of Chromatography Library, Elsevier, 137 (2002).

8. L. Lampugnani, C. Salvetti and D.L. Tsalev, Talanta 61, 683 (2003).

9. A. Thongsaw, W.C. Chaiyasith, R. Sananmuang, G.M. Ross and R.J. 
Ampiah-Bonney, Food Chem. 219, 453 (2017).

10. G.P. Brandão, R.C, de Campos and A.S. Luna, Spectrochim. Acta B 60, 625 (2005).

11. F.d.S. Dias, J.S. Bonsucesso, L.S. Alves, D.C.d.S. Filho, A.C.S. Costa and W.N.L.d. Santos, Microchem. J. 106, 363 (2013).

12. C. Vargas-Razo and J.F. Tyson, Fresenius J. Anal. Chem. 366, 182 (2000).

13. J.M., Mrmošanin, A.N. Pavlović, J.N. Krstic, S.S. Mitic, S.B. Tošic, M.B. Stojkovic, R.J. Mici and M.S. Dordevic, J. Food Compost. Anal. 67, 163 (2018).

14. B. Welz, M.G.R. Vale, D.L.G. Borges and U. Heitmann, Anal. Bioanal. Chem. 389, 2085 (2007).

15. A. Kabir, M. Locatelli and H.I. Ulusoy, Separations 4, 36 (2017).

16. M. Rezaee, Y. Assadi, M.R. Milani Hosseini, E. Aghaee, F. Ahmadi and S. Berijani, J. Chromatogr. A 1116 , 1 (2006).

17. H. Al-Saidi and A. Emara, J. Saudi Chem. Soc. 18, 745 (2014).

18. E.G. Primel, S.S. Caldas, L.C. Marube, A.L.V. Escarrone, Trends Environ. Anal. Chem. 14, 1 (2017).

19. M. Firat, S. Bakirdere, M.S. Fındikoglu, E.B. Kafa, E. Yazici, M. Yolcu, C.. Büyükpinar, D.S. Chormey, S. Sel and F. Turak, Spectrochim. Acta B 129, 37 (2017).

20. S. Erarpat, G. Özzeybek, D.S. Chormey and S. Bakirdere, Chemosphere 189, 180 (2017).

21. Q.Y. Yu, J.F. Huang, Y. Shen, L.M. Xiao, J.M. Liu, D.-B. Kuang and C.Y. Su, RSC Adv. 3, 19311 (2013).

22. C. Zhong, H. Huang, A. He and $\mathrm{H}$. Zhang, Dyes Pigm. 77, 578 (2008)

23. L. Yao, W. Xu, C. Lin, Y. Zhu, F. Luo, J. Zhang, H. Liu and L. Pang, Anal. Meth. 9, 4673 (2017).

24. S. Candir, I. Narin and M. Soylak, Talanta 77, 289 (2008).

25. H. Fu, X. Wang, Y. Sun, L. Yan, J. Shen, J. Wang, S.-T. Yang and Z. Xiu, Sep. Purif. Technol. 180, 44 (2017).

26. M. Chamsaz, A. Atarodi, M.
Eftekhari, S. Asadpour and M. Adibi, J. Adv. Res. 4, 35 (2013).

27. H. Xu, Y. Wu, J. Wang, X. Shang and X. Jiang, J. Environ. Sci. 25, S45 (2013).

28. V. Kazantzi, A. Kabir, K.G. Furton and A. Anthemidis, Microchem. J. 137, 285 (2018). 